\title{
High Resolution Prediction of Gas Injection Process Performance for Heterogeneous Reservoirs
}

\author{
Quarterly Report \\ April 1, 2001 to June 30, 2001 \\ Contract N0. DE-FC26-00BC15319 \\ Principal Investigator: Franklin M. Orr, Jr. \\ Department of Petroleum Engineering \\ Stanford University
}

\section{Disclaimer}

This report was prepared as an account of work sponsored by an agency of the United States Government. Neither the United States Government nor any agency thereof, nor any of their employees, makes any warranty, express or implied, or assumes any legal liability or responsibility for the accuracy, completeness, or usefulness of any information, apparatus, product, or process disclosed, or represents that its use would not infringe privately owned rights. Reference herein to any specific commercial product, process, or service by trade name, trademark, manufacturer, or otherwise does not necessarily constitute or imply its endorsement, recommendation, or favoring by the United States Government or any agency thereof. The views and opinions of authors expressed herein do not necessarily state or reflect those of the United States Government or any agency thereof. 


\section{Abstract}

This report outlines progress in the third 3 quarter of the first year of the DOE project "High Resolution Prediction of Gas Injection Process Performance for Heterogeneous Reservoirs." A simple theoretical formulation of vertical flow with capillary/gravity equilibrium is described. Also reported are results of experimental measurements for the same systems. The results reported indicate that displacement behavior is strongly affected by the interfacial tension of phases that form on the tie line that extends through the initial oil composition. 


\section{Contents}

1 Executive Summary $\quad 4$

2 Project Status $\quad 5$

3 Compositional Capillary/Gravity Equilibrium $\quad \mathbf{5}$

3.1 Theoretical Estimates of Gravity/Capillary Equilibrium . . . . . . . . . . . . . 5

3.2 Experimental Measurements of Compositional Flows . . . . . . . . . . . . . 8

4 Results and Discussion $\quad 9$

4.1 Experimental Results versus Theoretical Estimates . . . . . . . . . . . . . . 9

4.2 CT scanning . . . . . . . . . . . . . . . . . . . . . 13

5 Conclusion $\quad 14$ 


\section{Executive Summary}

Gas injection in oil reservoirs offers significant potential for improved oil recovery. However, successful design of a gas injection process requires a detailed understanding of a variety process mechanisms, including the phase behavior of multicomponent mixtures and the approach to multicontact miscibility in the reservoir, the flow of oil, water and gas in permable rocks, and the interaction of phase behavior, reservoir heterogeneity and gravity on overall performance at the field scale. This project examines these mechanisms using a combination of theoretical, numerical and laboratory studies of gas injection, as described below.

The aim of this work is to develop a set of ultra-fast compositional simulation tools that can be used to make field-scale predictions of the performance of gas injection processes. To achieve the necessary accuracy, these tools must satisfy the fundamental physics and chemistry of the displacement from the pore to the reservoir scales. Thus this project focuses on four main research areas:

1. Determination of the most appropriate methods of mapping multicomponent solutions to streamlines and streamtubes in $3 \mathrm{D}$,

2. Development of techniques for automatic generation of analytical solutions for onedimensional flow along a streamline,

3. Experimental investigations to improve the representation of physical mechanisms that govern displacement efficiency along a streamline, and

4. Theoretical and experimental investigations to establish the limitations of the streamline/streamtube approach. 


\section{Project Status}

In the report that follows briefly review the status of the research effort in each area. Ph.D. student Jichun Zhu and Professor Lynn Orr are in the processes of extending the analytical solutions of compositional flows to include variations in temperature. Postdoc Kristian Jessen is working on the automatic generation of analytical solutions. M.S. student Ashwini Upadhyaya is perfoming experiments on four-phase flow in micromodels and capillary tubes. M.S. student Linda Sam-Olibale is doing dimensional analysis and simulation to obtain the correct scaling parameters for cross-flow in heterogeneous systems. All of this research will be detailed in future reports. In this report, we concentrate on our progress in understanding the effect of capillary forces on compositional displacements, work performed by Research Associate Davis A. DiCarlo.

Gas injection processes are inherently compositional. Components from the initial oil phase will vaporize into the gaseous phase, and components of the injected gas will dissolve into the oil. In the past decade we have made major progress in understanding and modeling these displacements along a streamline. In this report we focus on experimental and theoretical arguments to examine how capillary and gravitational forces interact when the compositional changes are taking place.

The partitioning of components between the nonwetting and wetting phases during a displacement can be described as a displacement path. This path is the route that the compositions follow through a space of dimension nc-1 (nc is the number of components)from the initial oil composition to the injected gas composition. In multicomponent systems, this path may be quite complex, involving many shocks and rarefactions from tie line to tie line. Because of these jumps across tie lines the interfacial tension between the phases can be changing by orders of magnitude. Previous studies have shown that if the interfacial tension is low, effective gravity-driven displacements can take place [5]. In this study we attempt to determine which is the controlling interfacial tension and thus the appropriate dimensionless numbers to scale capillary forces for compositional displacements.

\section{Compositional Capillary/Gravity Equilibrium}

\subsection{Theoretical Estimates of Gravity/Capillary Equilibrium}

The set of equations that describe gravity/capillary equilibrium for a compositional displacement is as follows. The capillary pressure $P_{c}$ depends on the wetting-phase saturation $S_{w}$ through

$$
P_{c}=\sigma\left(x_{i}\right) J^{*}\left(S_{w}\right),
$$

where $x_{i}$ are the volume fractions and determine which tie line connects the nonwetting and wetting phases, and subsequently the interfacial tension, $\sigma$, while $J^{*}$ is the Leverett J-function for the particular porous medium.

The nonwetting and wetting phase pressures are obtained by setting the pressures equal to zero at the bottom of the column $(z=0)$. Then

$$
P_{w}=\int \rho_{w}\left(x_{i}\right) d z
$$




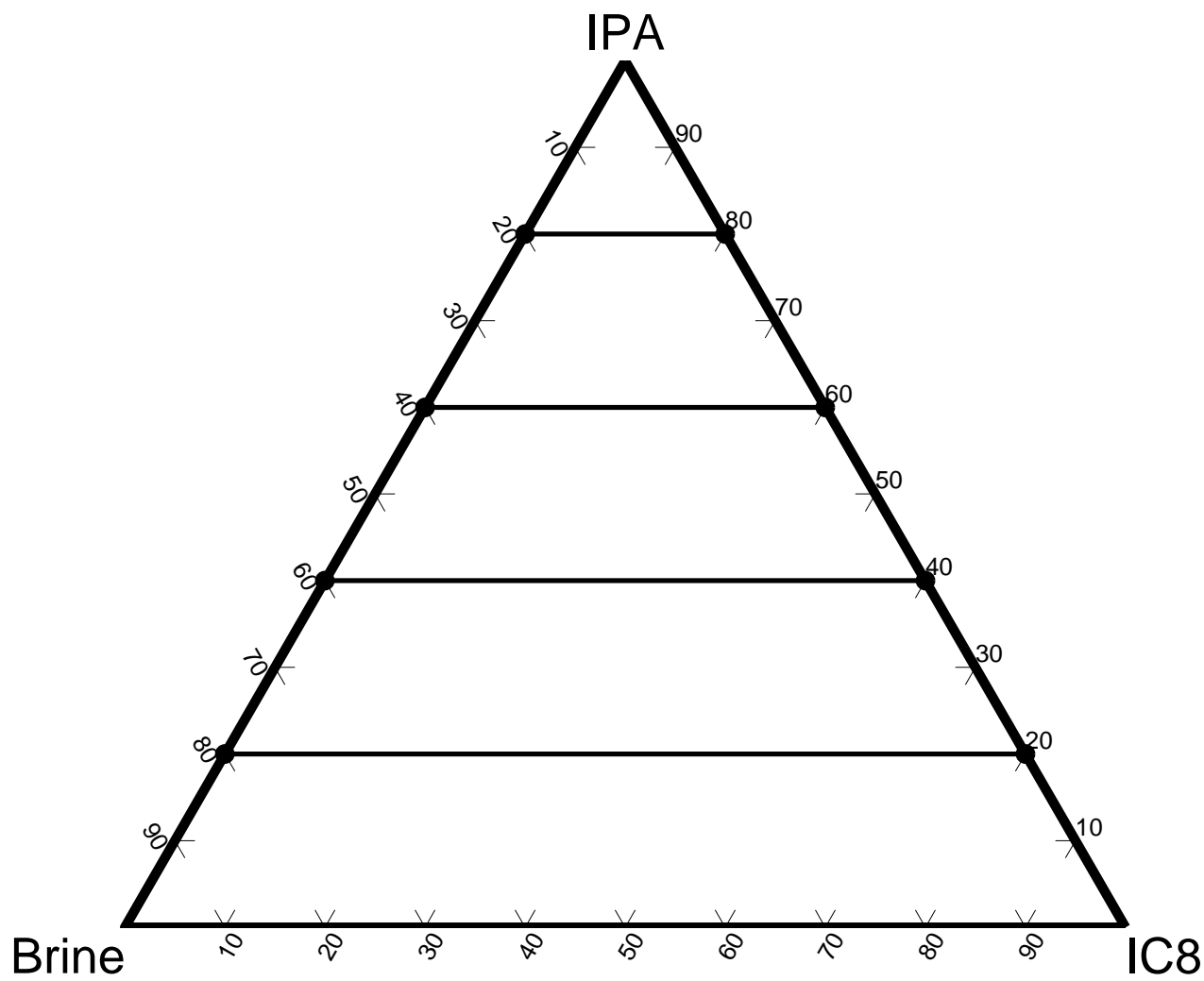

Figure 1: Simplified phase diagram for a two-phase three-component brine/isopropanol (IPA)/iso-octane (IC8) system.

$$
P_{n}=\int \rho_{n}\left(x_{i}\right) d z
$$

To close this set of equations, the conservation of each component must be added. Although this set of equations can be intractable for systems with many components and arbitrary phase behavior, it can be solved exactly for the simplified ternary oil, brine, and alcohol system shown in Fig. 1.

For this phase diagram, the tie lines are all parallel to the iso-octane/brine (O-B) axis, and all extend to the alkohol/iso-octane (A-O) and alcohol/brine (A-B) axes. Thus there is always a wetting and nonwetting phase. This is equivalent to having constant $\mathrm{K}$ values of $K_{O}=0, K_{A}=1$, and $K_{B}=\infty$. We also assume no volume change on mixing. The lever rule gives the nonwetting $\left(S_{N}\right)$ and wetting $\left(S_{W}\right)$ phase saturations in terms of the compositions.

$$
\begin{aligned}
S_{N} & =x_{O} / x_{2} \\
S_{W} & =x_{B} / x_{2},
\end{aligned}
$$

where

$$
x_{2}=1-x_{A} .
$$

The initial wetting phase in the column will consist of $x_{B}=x_{2}^{i}, x_{A}=x_{A}^{i}$, and $x_{O}=0$, and the displacing nonwetting phase will consist of $x_{B}=0, x_{A}=x_{A}^{d}$, and $x_{O}=x_{2}^{d}$.

We take for the interfacial tension $\sigma$

$$
\sigma=\sigma_{0} x_{2}^{3}
$$


where $\sigma_{0}$ is the interfacial tension for no alcohol, and for the densities

$$
\begin{aligned}
\rho_{N} & =\rho_{O}+x_{A}\left(\rho_{A}-\rho_{O}\right) \\
\rho_{W} & =\rho_{B}+x_{A}\left(\rho_{A}-\rho_{B}\right) \\
\Delta \rho & =\rho_{w}-\rho_{N}=x_{2}\left(\rho_{B}-\rho_{O}\right)
\end{aligned}
$$

where $\rho_{i}$ are the pure phase densities, and we assume no volume change on mixing.

If the phases move only by convection, and the components are always in equilibrium, the flux of each component is

$$
\begin{aligned}
& u_{O}=x_{2} u_{N} \\
& u_{B}=x_{2} u_{W} \\
& u_{A}=x_{A}\left(u_{N}+u_{W}\right)
\end{aligned}
$$

Consider the alcohol component. Assuming no diffusion, the conservation equation for the alcohol is

$$
\phi \frac{\partial x_{a}}{\partial t}+\frac{\partial u_{a}}{\partial z}=0
$$

The total flux, $u=u_{W}+u_{N}$, is constant in space, thus Eq. 14 becomes

$$
\phi \frac{\partial x_{a}}{\partial t}+u \frac{\partial x_{a}}{\partial z}=0
$$

As $\phi$ and $u$ are constant in space, Eq. 15 inticates that all alcohol compositions propagate at the same velocity. Thus for constant initial and injection conditions, the final alcohol distribution must be equal to the initial alcohol composition $\left(x_{A}^{i}\right)$ up to a certain height $z^{*}$ above which it will equal the injection alcohol composition $\left(x_{A}^{d}\right)$. Importantly, this $z^{*}$ will be given by the total flux in the column, and that the total amount of wetting phase retained in the column is given by $z^{*}$. Thus the lower the $z^{*}$ value the better recovery of the wetting phase when equilibrium is reached.

Finally, notice that capillary forces will not smear the alcohol front. Capillary forces will leave brine above the height $z^{*}$ and drive oil below the height $z^{*}$.

With this simple distribution of alcohol, the calculation of the height $z^{*}$ and the wetting and nonwetting phase saturations is straightforward. As there are only two different alcohol compositions in the final profile, there are only two interfacial tensions, and two density differences. The capillary pressure is thus

$$
P_{c}=\left\{\begin{array}{ll}
\Delta \rho^{i} g z & z \leq z^{*} \\
\Delta \rho^{i} g z^{*}+\Delta \rho^{d} g\left(z-z^{*}\right) & z \geq z^{*}
\end{array} .\right.
$$

The total brine composition left in the column must equal $x_{2}^{i} z^{*}$ due to the above conservation arguments. This must be equal to the amount of brine from the saturation profile. Equating the two gives an equation for $z^{*}$,

$$
x_{2}^{i} z^{*}=x_{2}^{i} \int_{0}^{z^{*}} J^{-1}\left(P_{c} / \sigma^{i}\right) d z+x_{2}^{d} \int_{z^{*}}^{L} J^{-1}\left(P_{c} / \sigma^{d}\right) d z .
$$

where $J^{-1}$ is the inverse Leverett J-function. This transcendental equation can easily be solved numerically by a bisection routine. Once $z^{*}$ is found, the capillary pressures and component distributions are found directly from the J-functions. 


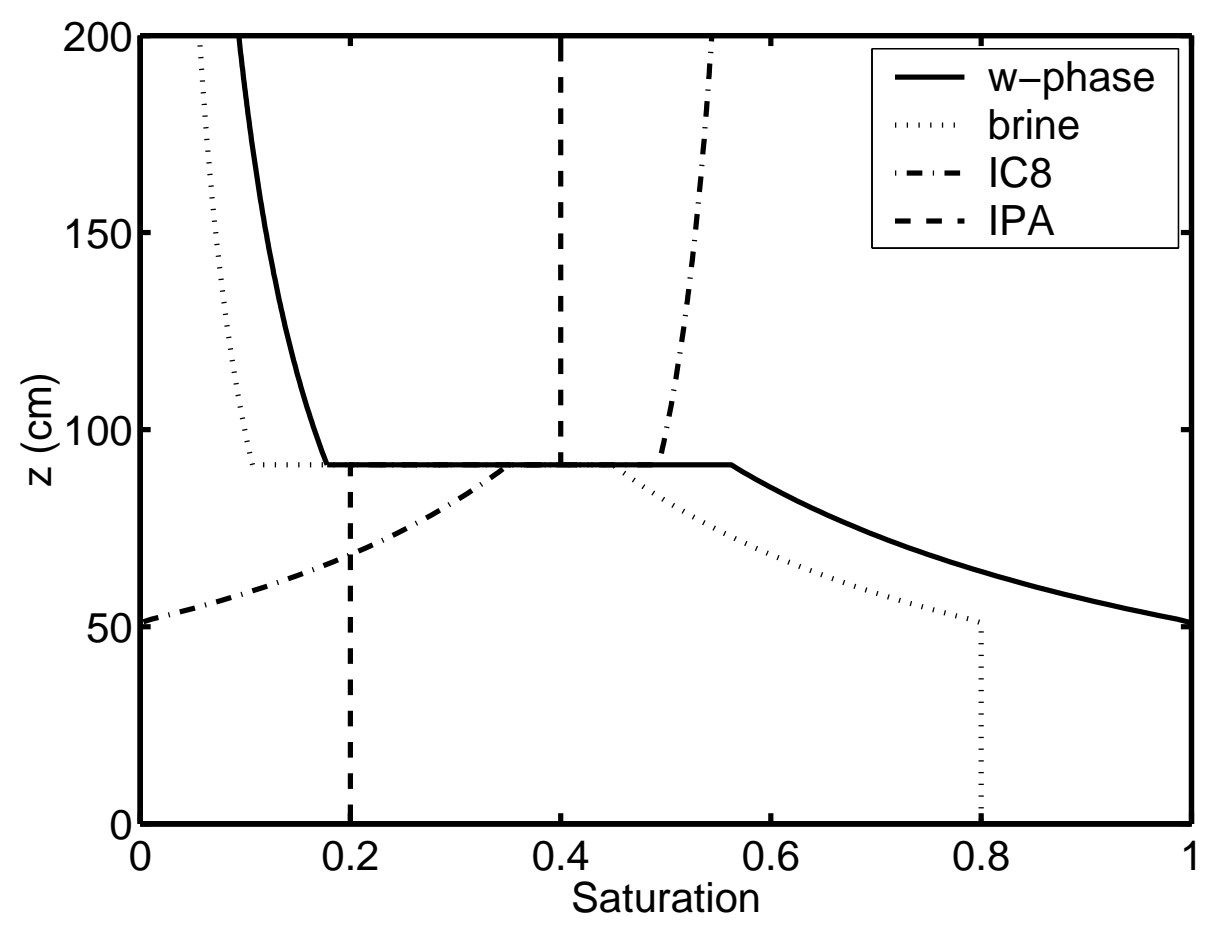

Figure 2: Vertical profile of the mass fraction compositions and wetting-phase saturation for a condensing drive at capillary/gravity equilibrium. For these particular parameters, the condensing drive displaces $55 \%$ of the initial oil $\left(z^{*}=90 \mathrm{~cm}\right)$.

Figure 2 shows the equilibrium distribution of each component for a condensing displacement with initial alcohol fraction of $x_{A}^{i}=0.2$ and displacing $x_{A}^{d}=0.4$. Figure 3 shows the equilibrium distribution of each component for a vaporizing displacement with initial alcohol fraction of $x_{A}^{i}=0.4$ and displacing $x_{A}^{d}=0.2$, the reverse of the condensing displacement. The $z^{*}$ height is lower for the condensing drive, and thus provides better overall recovery at gravity/capillary equilibrium. We find that the condensing displacement always has a better recovery than the vaporizing displacement when the alcohol compositions are reversed.

An interesting observation in these solutions is that for the condensing drive the brine composition is always decreasing with height, while for the vaporizing drive the brine composition increases with height when the alcohol composition decreases. This will be important when we compare these ideal gravity/capillary equilibrium solutions to what is observed in laboratory experiments.

\subsection{Experimental Measurements of Compositional Flows}

We performed laboratory experiments of these compositional drainages using an ana$\log$ compositional system consisting of isopropanol (IPA), iso-octane $\left(\mathrm{IC}_{8}\right)$, and a $10 \% \mathrm{NaBr}$ brine. This is similar with the system developed by Morrow et al.[2], except for the more concentrated brine. The room temperature/room pressure phase diagram has been measured and is depicted in Fig. 4. In this system, the iso-octane represents the light hydrocarbons, and is the primary constituent of the nonwetting (gaseous) phase. The brine represents 


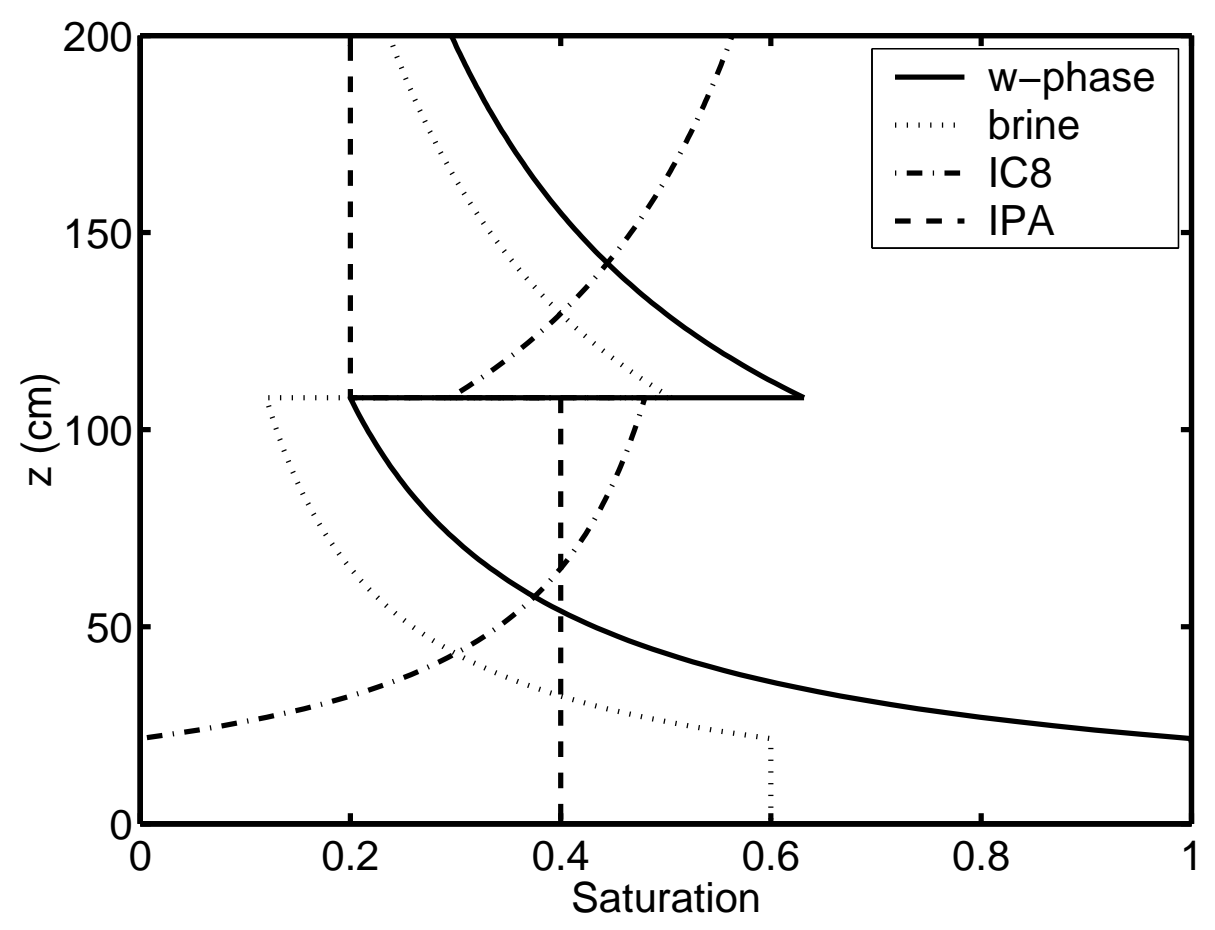

Figure 3: Vertical profile of the mass fraction compositions and wetting-phase saturation for a vaporizing drive at capillary/gravity equilibrium. For these particular parameters, the vaporizing drive displaces $45 \%$ of the initial oil $\left(z^{*}=110 \mathrm{~cm}\right)$.

the heavy hydrocarbons, and is the primary constituent of the wetting (liquid) phase. The isopropanol represents the intermediate hydrocarbons, and can transfer between the wetting and nonwetting phases.

The experiments were done in $60 \mathrm{~cm}$ long columns of size 60 sand. The columns consisted of 20 separate $3 \mathrm{~cm}$ long sections of polycarbonate tubing $(2.54 \mathrm{~cm}$ diameter which were held together with teflon shrink tubing. The column was flushed with $\mathrm{CO}_{2}$ and then filled with the working wetting phase, until all the gas bubbles dissolved. The primary drainage then took place by attaching a burette full of the working nonwetting phase to the top of the column. The bottom boundary condition of the wetting phase was at constant head, and the top boundary condition was with a slightly falling head. The change in the top boundary condition was typically $3 \mathrm{~cm}$ of oil. The heads were chosen such that no nonwetting phase would enter the wetting phase exit.

Each drainage ran for roughly 2 weeks, after which the column was sectioned every 3 $\mathrm{cm}$, and the components dissolved in ethanol and analyzed on a gas chromatograph.

\section{Results and Discussion}

\subsection{Experimental Results versus Theoretical Estimates}

Figures 5 and 6 show the final compositional profile and composition path for the condensing drive, while Figs 7 and 8 show the same data for the equivalent vaporizing drive. 


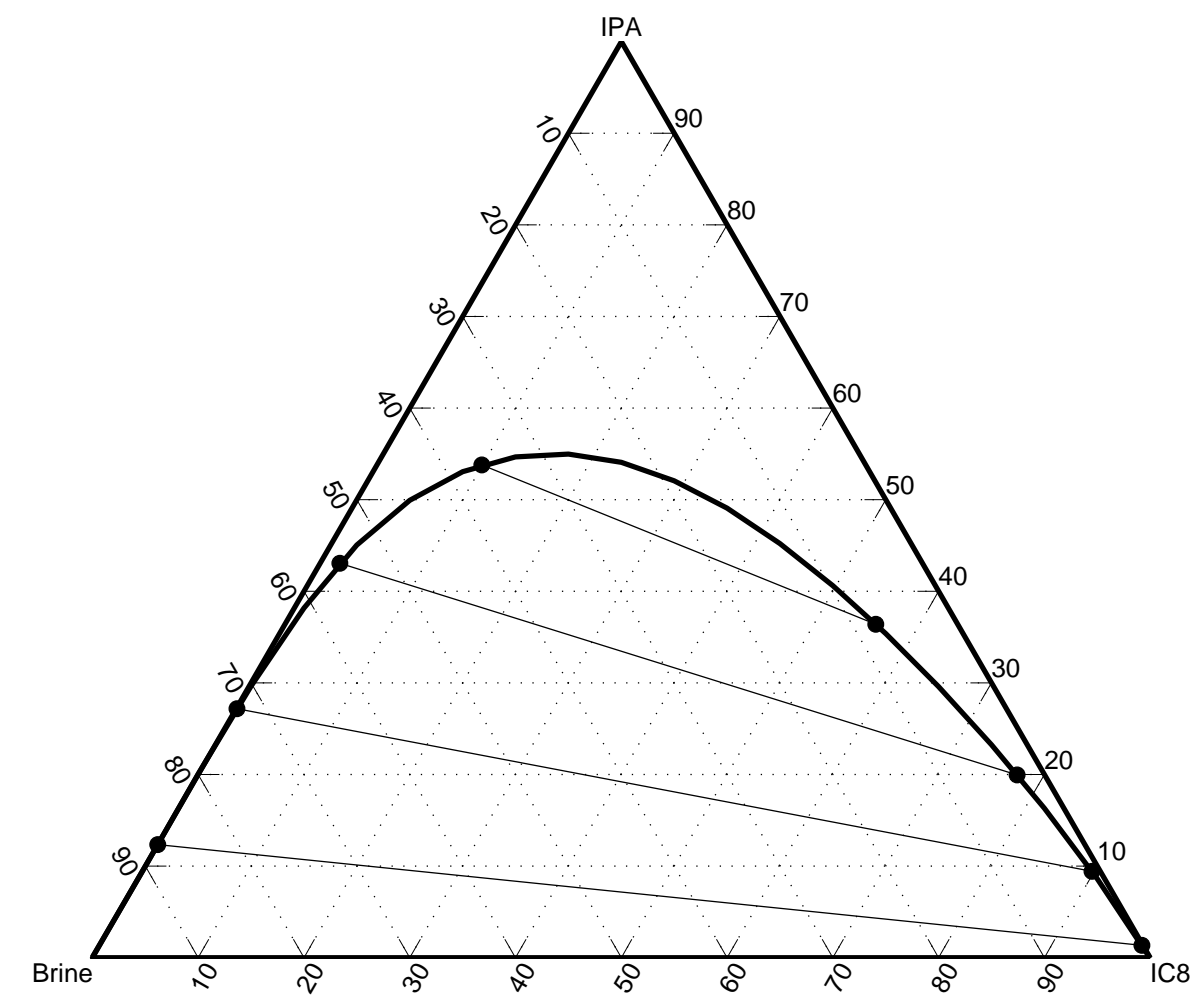

Figure 4: Phase diagram for the brine/isopropanol (IPA)/iso-octane (IC8) ternary system at room temperature and pressure.

The vaporizing drive produces much more of the wetting phase than the condensing drive. If we put the results in terms of $z^{*}$ 's we see that for the condensing drive $z^{*}=15 \mathrm{~cm}$ and for the vaporizing drive $z^{*}=-1 \mathrm{~cm}$. This is in marked contrast to the equilibrium solution for the simplified phase diagram, which showed that better recovery was found for the condensing drive.

Additionally, the paths in the composition space show that for the condensing drive there are only two compositions in large regions of the column, the initial nonwetting phase with a little residual brine at the top of the column and the initial wetting phase at the bottom. The transition is abrupt. For the vaporizing drive, there are three composition states. From top to bottom, the first region is the initial nonwetting phase with a little residual brine followed by a composition along the initial wetting-phase tie line, but nearer the nonwetting side and finally the initial wetting phase.

The results can be understood in terms of the equilibrium position being unobtainable, and the particular fanning of the tie lines for this system. The final compositional profile in the vaporizing measurement shows a region of low interfacial tension at the bottom of the column and high interfacial tension at the top. Thus there are capillary forces which act to pull the wetting phase toward the top of the column. As can be seen from the analytical results, equilibrium is not reached until an appreciable amount of wetting phase is pulled into this region. The experiments show that this does not occur on the time scale of the experiment. This is not surprising as the flow must be counter-current (wetting phase moving upwards and nonwetting phase moving downwards), and the wetting phase saturation in the regions at the top is low. Thus the relative permeabilites can be extremely small, and 


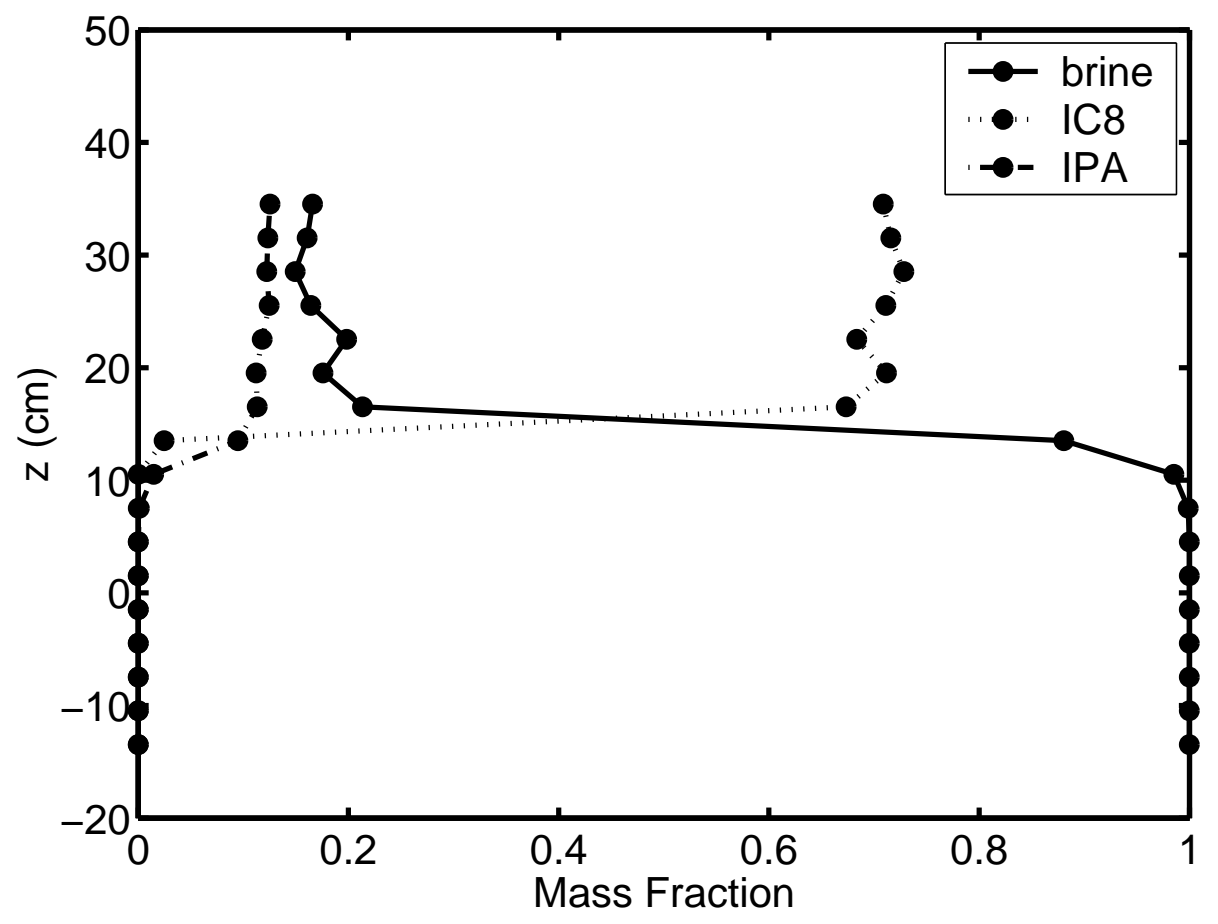

Figure 5: Composition profile for the condensing drive. The nonwetting phase does not enter below a height of $15 \mathrm{~cm}$ above the zero capillary pressure height.

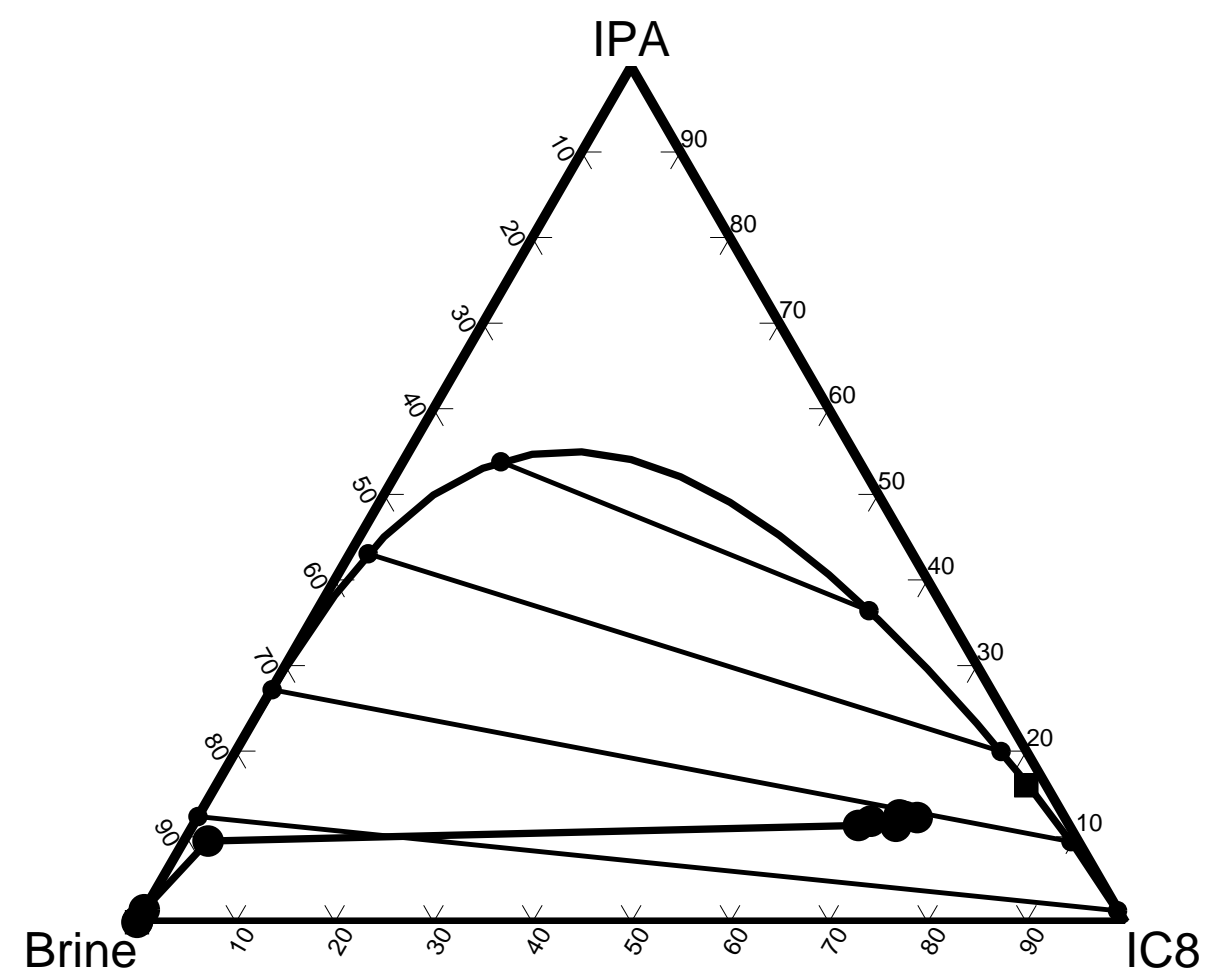

Figure 6: Composition path for the condensing drive. The squares are the initial "gas" and "oil" compositions. The column shows basically two compositions, the intial "oil" and the injected "gas" with a residual amount of brine. 


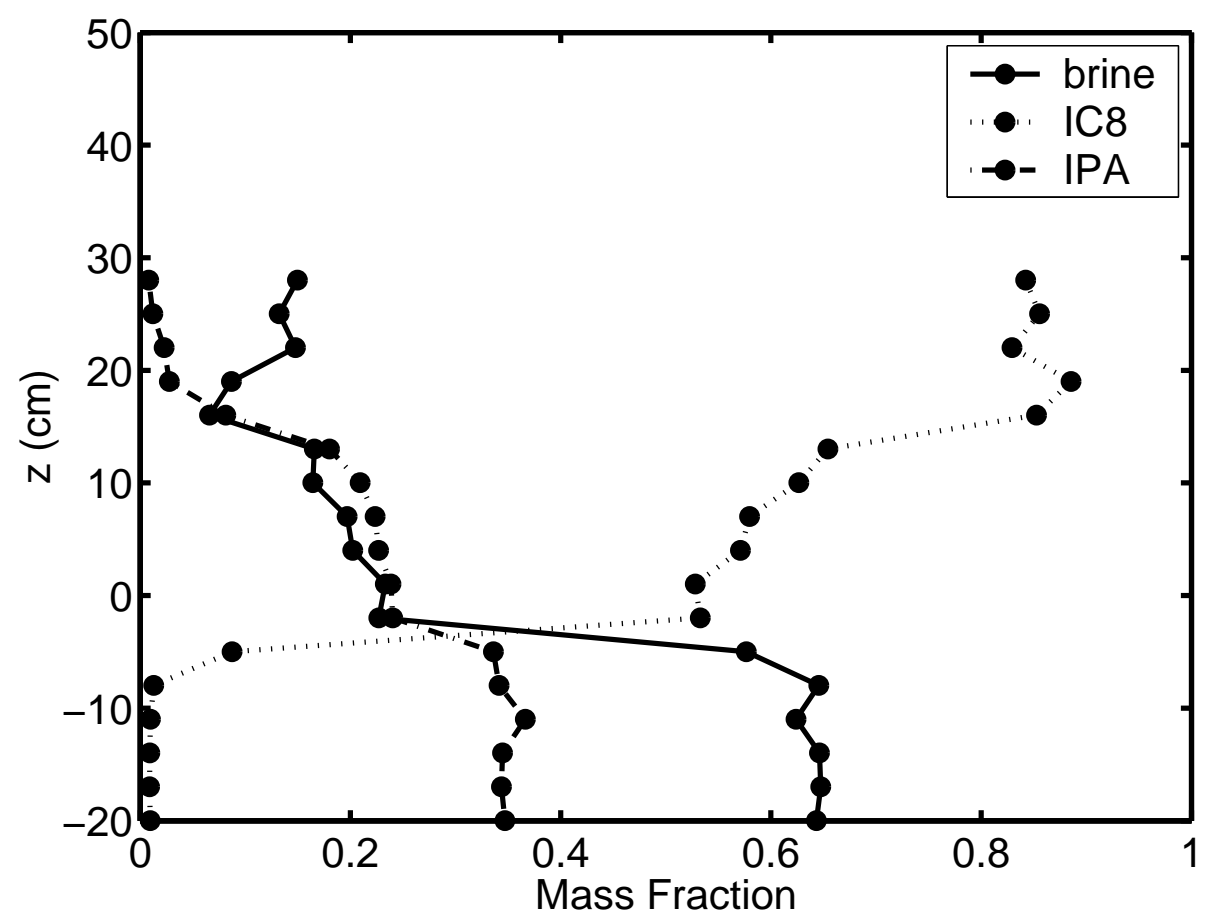

Figure 7: Composition profile for the vaporizing drive. The nonwetting phase enters below a height of $0 \mathrm{~cm}$ above the zero capillary pressure height, yieldiing a much better dispalcement than the condensing drive.

although there exists a capillary driving force, the flux moved by it is too small to allow equilibration in the time available.

In addition, these results show that the jumps into the two-phase region are along the tie lines even for gravitationally driven displacements, as is expected when advective forces dominate, and compositional equilibrium can be assumed. Three composition states in the vaporizing and two composition states in the condensing are a result of the dilution line being more parallel to the tie lines in the vaporizing case than in the condensing case. For mass conservation the measured compositions have to spend equal time on both sides of the dilution line. This is easily achieved for the more parallel vaporizing drive, but not for the less parallel condensing drive. Also for the condensing drive, the high interfacial tension of the initial fluid makes a jump into the two-phase region along this tie line impossible as the capillary forces holding the wetting phase in are large. Thus since advective and capillary forces permit no compositions below the dilution line, no compositions above the dilution line can be reached, and we see a shock from the initial to the final composition. In contrast for vaporizing drives, the high interfacial tension tie line is the injection tie line, which retains wetting phase leading to compositions below the dilution lines. This allows the state along the initial tie line to be reached (along with the small angle between the dilution line and the tie line), and three composition states are observed. 


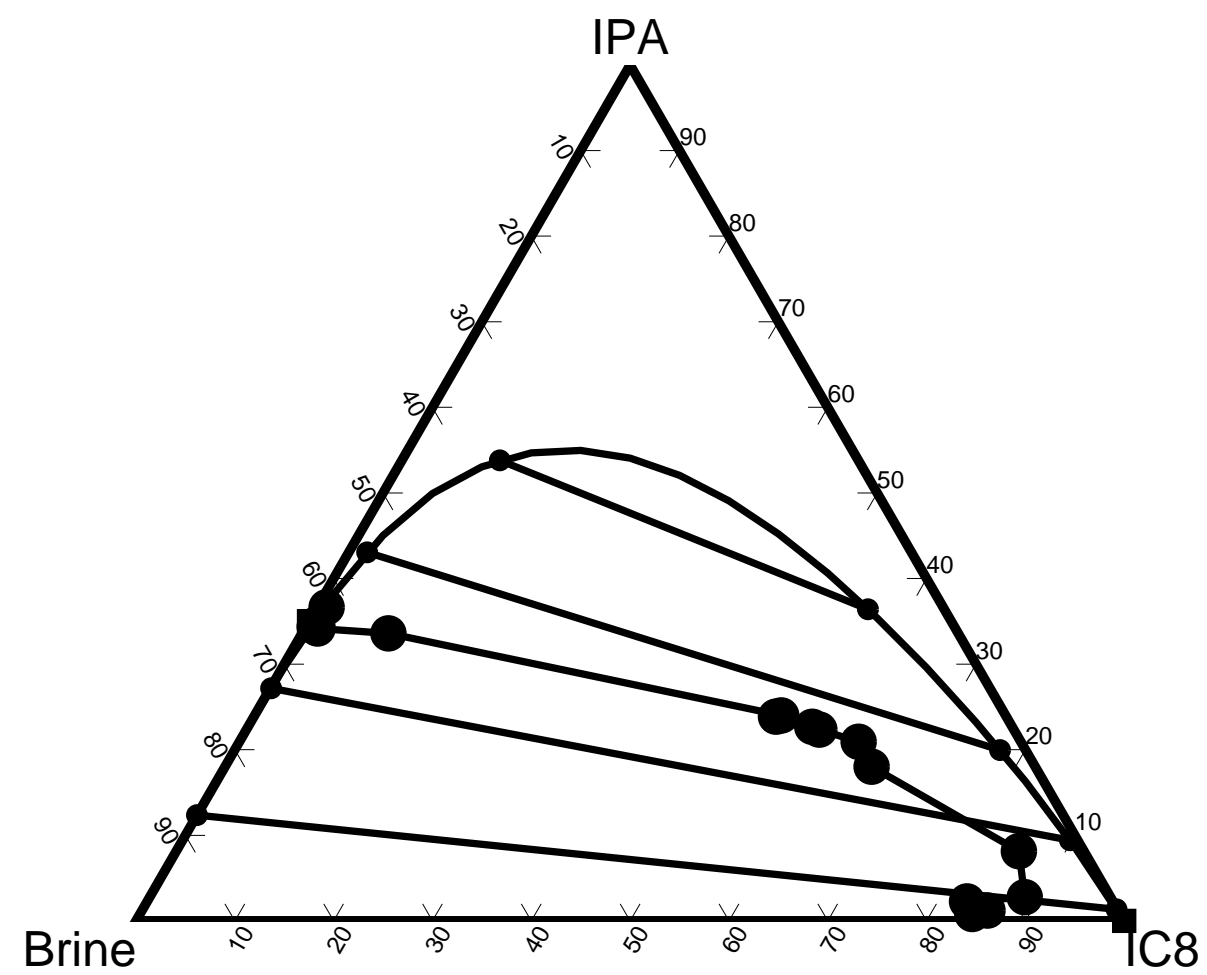

Figure 8: Composition path for the vaporizing drive. The squares are the initial "gas" and "oil" compositions. The column shows 3 compositions, the intial "oil", a state along the "oil" tie line with residual brine and the injected "gas" with a residual amount of brine.

\subsection{CT scanning}

CT scanning has been used widely to image in-situ saturation in space and time within a porous medium $[1,4]$. Here, we use CT scanning to image the composition profiles within columns similar to those used in the destructive experiments. This allows us to observe how the profiles change in space and time and to more accurately determine the physical mechanism that controls the movement of the phases. 


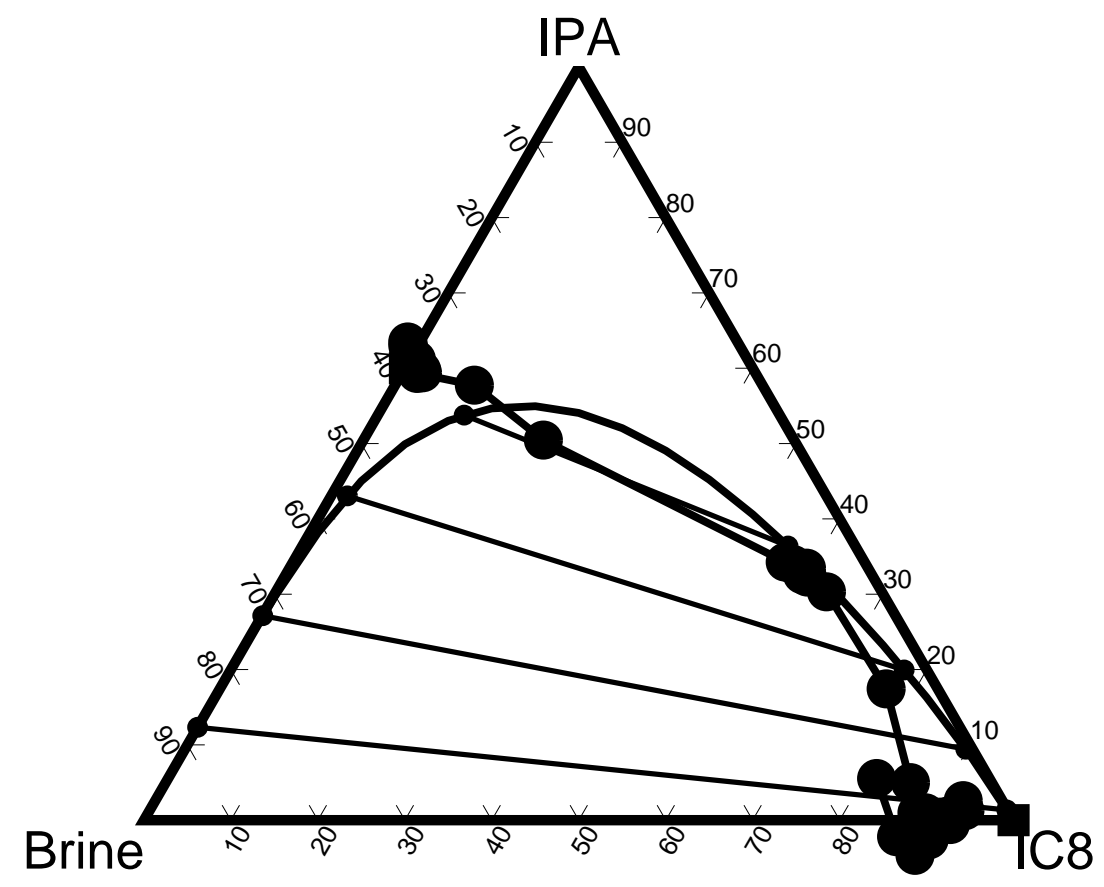

Figure 9: Composition path for the vaporizing drive. The results show the same behavior as seen in the column experiments and show that the CT can resolve the compositions as well as destructive methods.

The porous medium that we image consists of homogeneous sand pack with a diameter of $7.5 \mathrm{~cm}$ and a length of $65 \mathrm{~cm}$. We obtain images every $2 \mathrm{~cm}$ in the vertical direction. If the scans are uniform in the scan plane, we can determine the compositions at each vertical position and time with a mass fraction accuracy of \pm 0.01 for the brine component and \pm 0.05 for the alcohol and oil components. Details of the calibration and accuracy calculations can be found in an earlier DOE report [3].

For the vaporizing displacement, Fig. 9 shows the composition path after 30 days of drainage. The path through the composition space is equivalent to that seen in the column experiments. These results show a) that the CT scanner can be used to measure in-situ composition changes, and $b$ ) that there is no large scale movement of wetting phase upward over longer time scales.

\section{Conclusion}

In conclusion, the controlling interfacial tension (IFT) appears to be the IFT of the initial oil. Much better gravity drainage is observed when the IFT of the initial oil is low than when it is high, even when the injected gas has a high IFT. Although this is in contrast to the theoretical equilibrium results, this can be understood as the equilibrium results are never reached in real situations due to the low relative permeabilities during the required countercurrent flow. 


\section{References}

[1] DiCarlo, D.A., Sahni, A., and Blunt, M.J.: "The Effect of Wettability on Three-Phase Relative Permeability," Transport in Porous Media (2000) 39, 347-366.

[2] Morrow, N.R., Chatzis, I. and Taber, J.J.: "Entrapment and Mobilization of Residual Oil in Bead Packs," Soc. Pet. Eng. Res. Eng. (1988) 3, 927-935.

[3] Orr, F.M., Jr., and Hewett, T.: "High Resolution Prediction of Gas Injection Process Performance for Heterogeneous Reservoirs," Quarterly Report No. 1, U.S. DOE Grant No. DE-FC26-00BC15319, U.S. Department of Energy, Bartlesville, OK (2000).

[4] Sahni, A., Burger, J.E. and Blunt, M.J.: "Measurement of Three Phase Relative Permeability during Gravity Drainage using CT Scanning," paper SPE 39655 in the proceedings of the 1998 SPE/DOE Improved Oil Recovery Symposium, Tulsa, OK, April.

[5] Schechter, D.S., Zhou, D. and Orr, F.M., Jr.: "Low IFT Drainage and Imbibition," J. Pet. Sci. Eng. (1994) 11, 283-300. 